\title{
Guidelines
}

Respirailion

\section{Idiopathic Pulmonary Fibrosis in Switzerland: Diagnosis and Treatment}

\author{
Position Paper of the Swiss Working Group for Interstitial and Rare Lung Diseases of the \\ Swiss Respiratory Society
}

\author{
Manuela Funke-Chambour ${ }^{a}$ Andrea Azzolab Dan Adler $^{c}$ \\ Constance Barazzone-Argiroffo ${ }^{d}$ Christian Benden ${ }^{e}$ Annette Boehler ${ }^{f}$ \\ Pierre-Olivier Bridevaux ${ }^{g}$ Martin Brutsche ${ }^{\text {h }}$ Christian F. Clarenbach ${ }^{\mathrm{e}}$ \\ Katrin Hostettler ${ }^{i}$ Rebekka Kleiner-Fingerg Laurent P. Nicod ${ }^{j}$ Paola M. Soccalc \\ Michael Tamm ${ }^{i}$ Thomas Geiser ${ }^{\mathrm{a}}$ Romain Lazor ${ }^{j}$ \\ ${ }^{a}$ Department of Pulmonary Medicine, Inselspital, Bern University Hospital, University of Bern, Bern, ${ }^{b}$ Pulmonology \\ Service, Department of Internal Medicine, EOC Hospital Civico Lugano, Lugano, 'Division of Pulmonary Diseases, \\ Department of Medical Specialties, and d Pediatric Pulmonology Unit, Department of Pediatrics, HUG, Geneva \\ University Hospitals, Geneva, e Division of Pulmonology, University Hospital Zurich, and f University Hospital Zurich, \\ Zurich, ${ }^{9}$ Department of Pulmonology, Hôpital du Valais, Sion, ${ }^{\text {h}}$ Department of Pulmonology, Cantonal Hospital St. \\ Gallen, St. Gallen, 'Clinic of Respiratory Medicine, University Hospital Basel, Basel, and 'Department of Pulmonology, \\ CHUV, University Hospital Lausanne, Lausanne, Switzerland
}

\section{Keywords}

Idiopathic pulmonary fibrosis · Nintedanib · Pirfenidone ·

Pulmonary fibrosis · Interstitial lung disease

\begin{abstract}
Idiopathic pulmonary fibrosis (IPF) is a severe progressive and irreversible lung disease. Novel antifibrotic drugs that slow disease progression are now available. However, many issues regarding patient management remain unanswered, such as the choice between available drugs, their use in particular subgroups and clinical situations, time of treatment onset, termination, combination or switch, or nonpharmacologic management. To guide Swiss respiratory physicians in this evolving field still characterized by numerous areas of
\end{abstract}

\section{KARGER}

(C) 2017 S. Karger AG, Basel

E-Mail karger@karger.com

www.karger.com/res uncertainty, the Swiss Working Group for interstitial and rare lung diseases of the Swiss Respiratory Society provides a position paper on the diagnosis and treatment of IPF.

(c) 2017 S. Karger AG, Basel

\section{Introduction}

Idiopathic pulmonary fibrosis (IPF) is a severe progressive lung disease. There is no available treatment to reverse the fibrotic process $[1,2]$. With recent publications of positive phase III studies and approval of novel

\section{T. Geiser and R. Lazor share last authorship.}

Dr. Manuela Funke-Chambour

Department of Pulmonary Medicine, Inselspital, Bern University Hospital University of Bern, Freiburgstrasse 8

CH-3010 Bern (Switzerland)

E-Mail Manuela.funke-chambour@insel.ch 
antifibrotic drugs to slow lung function decline, several national and international respiratory societies have decided to update existing guidelines or endorse new international guidelines [3-5]. The antifibrotic drugs pirfenidone and nintedanib are now recommended for patients with IPF according to the latest ATS/ERS/JRS/ALAT guidelines [2]. Yet, many questions regarding the management of IPF patients remain the subject of debate [2], for instance, when to start and when to stop treatment.

Now that nintedanib and pirfenidone have been approved by the Swiss health authorities (SwissMedic) and they are available for prescription, the Swiss Working Group for interstitial and rare lung diseases of the Swiss Respiratory Society decided to formulate a position paper to guide physicians in Switzerland in the diagnosis and treatment of IPF. In addition, due to the different languages used in Switzerland, Swiss physicians often refer to guidelines in their mother tongue, which might lead to different treatment approaches in one country. Thus, German and French recommendations have been compared with the current international guidelines to establish a national recommendation for Switzerland.

\section{Methodology}

The recommendations in this position paper are based on the current international literature and opinion of the members of the Swiss Working Group for interstitial and rare lung diseases of the Swiss Respiratory Society (A.A., D.A., C.B.-A., A.B., P.-O.B., M.B., C.C., M.F.-C., K.H., R.L., R.K.-F., and T.G., hereafter called the "working group").

A core panel was constituted with all members of the working group. A review panel was constituted by adding selected experts to the core panel (C.B., L.P.N., and P.M.S.). As a first step, a list of questions to be addressed was established (M.F.-C., T.G., and R.L.) and was distributed for review. Using an electronic survey tool, we classified the relevance of each question as "very important," "important," "of limited interest," or "unimportant," rated between 1 = "fully disagree" and 9 = "fully agree." Individual votes are represented in the percentage of votes for each grade. Questions considered by the majority as "very important" and "important" were reviewed, discussed, and reformulated if needed. A standardized wording was defined to express the strength of the recommendations: the terms "we recommend" or "should" were used to express a strong recommendation; the terms "we suggest" or "may" were used to express a weak recommendation; the terms "we do not recommend" or "should not" were used to express negative recommendations.

A first draft of possible responses was written (M.F.-C. and T.G.) and discussed in a face-to-face meeting. A second version was then established (M.F.-C., T.G., and R.L.) and redistributed for review. An iterative formal consensus technique (Delphi method) [6] was then used to measure and improve consensus within the group, using an electronic survey tool. In each round, the members
Table 1. Interpretation of the consensus level [6]

\begin{tabular}{ll}
\hline $\begin{array}{l}\text { Perfect } \\
\text { consensus }\end{array}$ & All respondents agree on an answer \\
\hline $\begin{array}{l}\text { Very good } \\
\text { consensus }\end{array}$ & $\begin{array}{l}\text { The median and middle } 50 \% \text { (interquartile } \\
\text { range) of the respondents are found at } 1 \text { interval } \\
\text { (e.g., the median and interquartile range are } \\
\text { both at } 8 \text { ) or } 80 \% \text { of the respondents are within } \\
1 \text { interval of the median (e.g., the median is } 8, \\
\text { 80\% of the respondents are between } 7 \text { and 9) }\end{array}$ \\
\hline $\begin{array}{l}\text { Good } \\
\text { consensus }\end{array}$ & $\begin{array}{l}\text { 50\% of the respondents are within } 1 \text { interval of } \\
\text { the median (e.g., the median is } 8,50 \% \text { of the } \\
\text { respondents are between } 7 \text { and } 9 \text { ) or } 80 \% \text { of the } \\
\text { respondents are within } 2 \text { intervals of the median } \\
\text { (e.g., the median is 7, } 80 \% \text { of the respondents } \\
\text { are between } 5 \text { and 9) }\end{array}$
\end{tabular}

Some $\quad 50 \%$ of the respondents are within 2 intervals of

consensus the median (e.g., the median is $7,50 \%$ of the respondents are between 5 and 9 ) or $80 \%$ of the respondents are within 3 intervals of the median (e.g., the median is $6,80 \%$ of the respondents are between 3 and 9)

No consensus All other responses

were asked to express their level of agreement with each recommendation, using a 7-point Likert scale, and to provide comments as needed. For divergent points, the text was amended and recirculated for review. The Swiss recommendations of the working group are marked with gray shading in the text. The final level of agreement was expressed using the predefined wording (Table 1). A summary of international, French, German, and Swiss recommendations is given in Table 2 for comparison.

\section{What Is IPF?}

\section{Clinical Presentation and Pathophysiology}

of the Disease

IPF is a fibrosing process of the lung parenchyma usually resulting in a restrictive pattern on pulmonary functional testing and impaired gas exchange, eventually leading to respiratory failure. Patients usually present with unspecific respiratory symptoms such as dyspnea on exertion and dry cough [1]. The pathophysiology of IPF has been extensively investigated. The disease is now considered to be due to repeated microinjuries to the alveolar epithelium caused by unknown agents and an impaired wound healing response, which leads to impairment of the lung's physiology [7].

Research efforts at the cellular and molecular levels have shown that multiple cytokines and mediators are 
Table 2. Overview of international, French, German, and Swiss recommendations

\begin{tabular}{|c|c|c|c|c|c|}
\hline \multicolumn{6}{|l|}{ Comparison of guidelines for idiopathic pulmonary fibrosis } \\
\hline & ATS 2011 & German 2013 & French 2013 & ATS 2015 & Swiss 2017 \\
\hline Method & grade & grade & recommendations & grade & recommendations \\
\hline \multicolumn{6}{|l|}{ Assessment } \\
\hline Bronchoalveolar lavage cellular analysis & 2 & 3 & + & na & 4 \\
\hline Transbronchial biopsy & 2 & 2 & na & & 2 \\
\hline Serology (RF, anti-CCP, ANA) & 3 & 3 & + & & 4 \\
\hline MDD & 4 & 4 & + & & 4 \\
\hline Genetics & - & - & + & & 2 \\
\hline Site of biopsy & na & $\begin{array}{l}\text { defined by MDD, } \\
\text { no area with exclusive } \\
\text { honeycombing }\end{array}$ & na & & $\begin{array}{l}\text { defined by MDD, } \\
\text { no area with } \\
\text { exclusive } \\
\text { honeycombing } \\
\end{array}$ \\
\hline Lung functional tests & na & na & $\begin{array}{l}\text { FVC, DLCO, } \\
\text { blood gas, } \\
\text { 6-MWT, Bioxx }\end{array}$ & & 3 \\
\hline \multicolumn{6}{|l|}{ Treatment and prevention } \\
\hline Acetylcysteine monotherapy & 2 & 3 & + & 2 & 1 \\
\hline Ambrisentan & na & na & - & 1 & na \\
\hline Anticoagulation & 2 & 1 & - & 1 & \\
\hline Azathioprine, acetylcysteine, prednisone & 2 & 1 & - & 1 & 1 \\
\hline Bosentan & 1 & 1 & - & 2 & \\
\hline Colchicine & 1 & 1 & - & na & \\
\hline Combined corticosteroids and immunomodulator therapy & 1 & 1 & na & na & \\
\hline Corticosteroid monotherapy & 1 & 1 & - & na & \\
\hline Corticosteroids for acute exacerbation & 3 & 3 & + & na & 3 \\
\hline Cyclosporine A & 1 & 1 & - & na & \\
\hline Etanercept & 1 & 1 & - & na & \\
\hline Imatinib & $\begin{array}{l}\text { no recommen- } \\
\text { dation }\end{array}$ & 1 & na & 1 & \\
\hline Interferon- $\gamma 1 \mathrm{~b}$ & 1 & 1 & - & na & \\
\hline Low-dose corticosteroids for treatment-resistant cough & na & na & + & na & na \\
\hline Lung transplantation & 4 & 4 & + & $\begin{array}{l}\text { no rec. single } \\
\text { vs. bilat. }\end{array}$ & 4 \\
\hline Macitentan & na & na & - & 2 & \\
\hline Invasive mechanical ventilation & 2 & 2 & - & na & 2 \\
\hline Nintedanib & na & na & na & 3 & 3 \\
\hline Oxygen therapy in hypoxic patients & 4 & 4 & + & na & 4 \\
\hline Palliative care with opioid treatment & $\begin{array}{l}\text { should be } \\
\text { considered }\end{array}$ & 3 & na & na & 4 \\
\hline Pirfenidone & $\begin{array}{l}\text { no recommen- } \\
\text { dation }\end{array}$ & 3 & + & 3 & 3 \\
\hline Pulmonary rehabilitation & 3 & 3 & + & na & 4 \\
\hline Sildenafil & $\begin{array}{l}\text { no recommen- } \\
\text { dation }\end{array}$ & na & na & 2 & \\
\hline Tobacco cessation & na & 3 & na & na & \\
\hline Treatment of asymptomatic reflux & 3 & $\begin{array}{l}\text { no recom- } \\
\text { mendation }\end{array}$ & + & 3 & 2 \\
\hline Treatment of pulmonary hypertension & 2 & $\begin{array}{l}\text { treatment trial if } \\
\text { severe with mild } \\
\text { fibrosis }\end{array}$ & only if severe & $\begin{array}{l}\text { no recommen- } \\
\text { dation }\end{array}$ & \\
\hline Vaccination against influenza and pneumococcus & na & 3 & + & na & na \\
\hline \multicolumn{6}{|l|}{ Follow-up } \\
\hline
\end{tabular}

4, strong recommendation for; 3 , weak recommendation for; 2 , weak recommendation against; 1 , strong recommendation against; +, recommendation for without weak or strong; -, recommendation against without weak or strong; "no recommendation," question has been addressed, but no recommendation has been formulated; na, not addressed. Transplantation: the Swiss recommendations are strong for considering transplantation. RF, rheumatoid factor; $\mathrm{CPP}$, cyclic citrullinated peptide; ANA, anti-nuclear antibodies; MDD, multidisciplinary discussion; FVC, forced vital capacity; DLCO, diffusing capacity of the lung for carbon monoxide; 6-MWT, 6-min walk test.

Idiopathic Pulmonary Fibrosis in Switzerland
Respiration 2017;93:363-378

DOI: $10.1159 / 000464332$ 
Table 3. Radiological criteria for UIP [1]

\begin{tabular}{|c|c|c|}
\hline UIP (all 4) & Possible UIP (all 3) & Inconsistent with UIP (any of 7) \\
\hline $\begin{array}{l}\text { Subpleural, basal predominance } \\
\text { Reticular abnormality } \\
\text { Honeycombing with or without traction } \\
\text { bronchiectasis } \\
\text { Absence of features listed as inconsistent } \\
\text { with UIP }\end{array}$ & $\begin{array}{l}\text { Subpleural, basal predominance } \\
\text { Reticular abnormality } \\
\text { Absence of features listed as inconsistent } \\
\text { with UIP }\end{array}$ & $\begin{array}{l}\text { Upper or mid-lung predominance } \\
\text { Peribronchovascular predominance } \\
\text { Extensive ground-glass abnormality } \\
\text { (extent > reticular abnormality) } \\
\text { Profuse micronodules } \\
\text { Discrete cysts } \\
\text { Diffuse mosaic attenuation/air trapping } \\
\text { Consolidation in bronchopulmonary } \\
\text { segment }\end{array}$ \\
\hline
\end{tabular}

UIP, usual interstitial pneumonia.

implicated in IPF pathogenesis, and transforming growth factor (TGF) $\beta$ was identified as a key player [8]. TGF- $\beta$, PDGF (platelet-derived growth factor), FGF (fibroblast growth factor), and VEGF (vascular endothelial growth factor) are profibrotic cytokines which are targets of current therapeutic approaches [8]. Excessive production of these and other cytokines leads to accumulation of differentiated fibroblasts, so-called myofibroblasts, in the alveolar interstitium. Myofibroblasts produce and modify the extracellular matrix (e.g., collagen), which alters the normal lung architecture and impairs gas exchange.

The histopathological features of IPF include fibroblast foci, which represent subepithelial accumulations of myofibroblasts in fibrotic areas with adjacent dense collagen deposits, and spatial and temporal heterogeneity of fibrotic changes. These changes constitute the so-called usual interstitial pneumonia (UIP) pattern. The radiological UIP pattern can be identified by high-resolution computed tomography (HRCT) if characteristic imaging features are present, and some correlations between histopathological changes and radiological features have been made previously (see below and Table 3) [1,9]. While an IPF diagnosis requires a radiological and histological UIP pattern (if histology has been performed), the presence of a UIP pattern does not necessarily implicate IPF, since it may occur in other contexts such as rheumatoid arthritis [10], chronic hypersensitivity pneumonitis [11], or connective tissue disease [12], which are important differential diagnoses to exclude.

Many factors have been found to increase the risk - or to contribute to the occurrence - of IPF, including old age [1], male gender [13], smoking [14], occupational exposures, gastroesophageal reflux [15-17], and viral or bacterial infections [18]. Genetic mutations (e.g., SPC, SPA, and telomerase genes) and single nucleotide polymorphisms (e.g., MUC5B) are increasingly identified in patients with IPF [19] and strongly suggest that a genetic susceptibility plays a role in disease development [19, 20].

The prevalence of IPF is described as increasing in some populations [21]. Data about the incidence and prevalence of pulmonary fibrosis vary due to changing case definitions in different countries [22]. An overview on interstitial lung disease (ILD) prevalence or incidence rates in Europe, including data about IPF, was given in a paper describing the epidemiology of different ILDs in Greece [23]. In Switzerland, there might be 100-5,000 IPF patients (considering previously published data), with an incidence of 18-1,424 new patients per year nationwide [8]. However, the true incidence and prevalence as well as the genetic characteristics of IPF in Switzerland are currently unknown.

\section{Course of the Disease}

Although new treatment options are now available, IPF remains a severe and life-threatening disease with a median survival of about 3 years $[1,24]$. The disease course can, however, differ considerably between individual patients. While some remain almost stable for years, others may progress rapidly or experience episodes of acute worsening or exacerbation. Acute exacerbation of IPF (AE-IPF) is an acute, clinically significant respiratory deterioration characterized by evidence of new, widespread alveolar abnormalities that typically occur within less than 1 month [25]. It is considered to be an independent acceleration of the disease process or response to occult external events $[25,26]$. The annual in- 

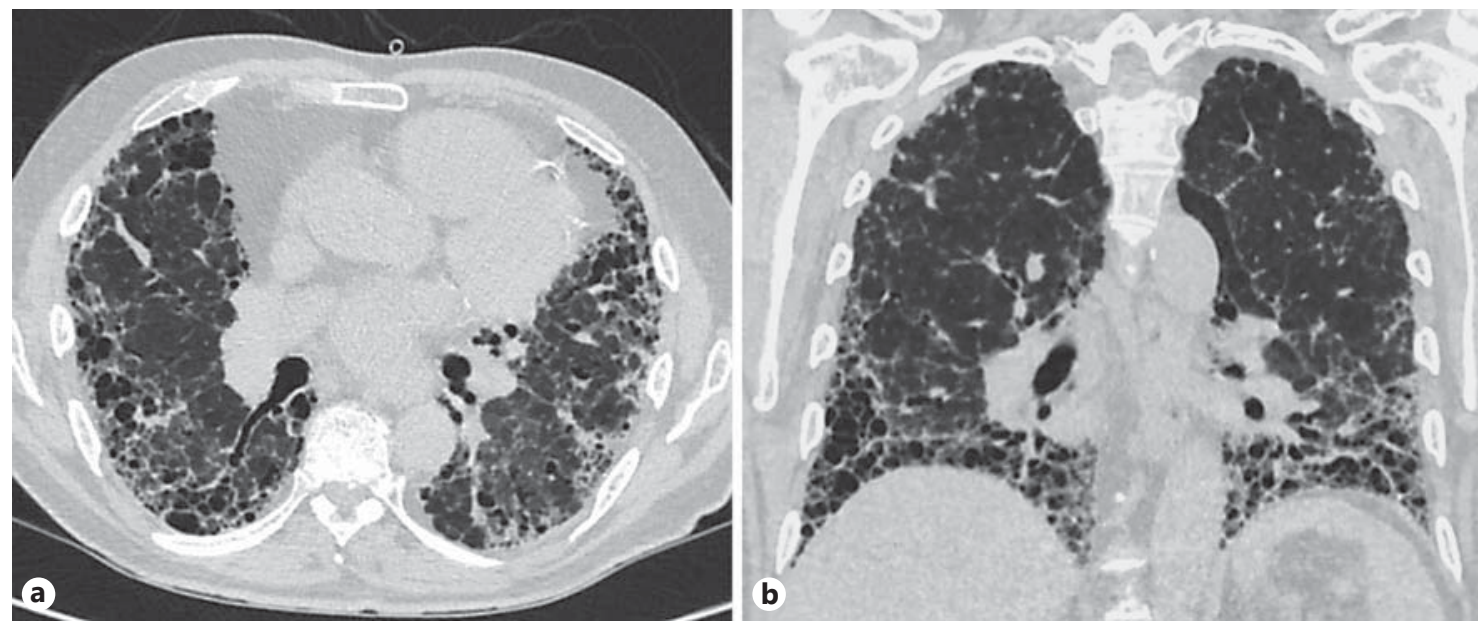

Fig. 1. Typical high-resolution computed tomography images of an idiopathic pulmonary fibrosis patient (a, b) with a basal gradient $(\mathbf{b})$, honeycombing, and traction bronchiectasis in the absence of a predominance of ground glass.

cidence of AE-IPF has been estimated around 5\% in placebo arms of recent randomized controlled trials, with a median survival of less than 3 months [27]. CT scanning during AE-IPF typically shows new ground-glass opacities superimposed on preexisting chronic fibrotic changes [26]. If biopsies are available, diffuse alveolar damage is usually present on the underlying UIP [26]. The definition of AE-IPF has recently been updated [25].

Exclusion of alternative causes - specifically heart failure as well as pulmonary embolism, pleural effusion, and pneumothorax - is crucial, as these causes affect the treatment approach and prognosis $[25,26]$. While exclusion of infection by bronchoalveolar lavage (BAL) or endotracheal aspirate has previously been required, it is no longer mandatory for the redefined term of "acute exacerbation" $[25,27]$. Nevertheless, infections need to be searched for, as they inform treatment decisions.

No reliable marker for predicting the disease course in IPF is available today, although MUC5B polymorphisms have been associated with better survival [28], and a variant of TOLLIP was related to higher mortality [29]. Today, only MUC5B polymorphisms and MMP7 are considered potential biomarkers of IPF disease course, even if no biomarker is used in clinical practice yet. [30] More commonly used in clinical practice are clinical and physiological variables (gender, age, forced vital capacity [FVC], and diffusing capacity of the lung for carbon monoxide [DLCO]) to predict mortality among patients with IPF, using various clinical scores such as the GAP index [31].

Idiopathic Pulmonary Fibrosis in Switzerland

\section{Diagnosis of IPF}

Current international guidelines require the exclusion of other known causes of ILD, the presence of a UIP pattern on HRCT if no surgical biopsy is obtained, or specific combinations of HRCT and surgical lung biopsy (SLB) if a biopsy is performed [1]. Most challenging is the exclusion of secondary causes of fibrosis. The initial step is thus a thorough patient history to exclude any environmental, occupational, or drug exposure to agents with profibrotic side effects. The patient history can point to connective tissue disease. The family history might reveal hereditary forms of pulmonary fibrosis, which is found in about $5 \%$ of cases [1]. History-taking should also include a search for possible triggering factors (e.g., gastroesophageal reflux) and comorbidities like obstructive sleep apnea or signs of coronary heart disease [1].

A clinical examination might reveal signs of chronic hypoxemia with finger clubbing and, eventually, cyanosis in advanced situations. Pulmonary auscultation usually reveals typical bibasilar Velcro crackles. Pulmonary functional tests may typically show a restrictive ventilatory defect with a reduced diffusion capacity and, possibly, hypoxia. If performed, exercise testing uncovers gas exchange impairment by desaturation during exercise [1].

According to international guidelines, a UIP needs to be present on HRCT for a diagnosis, which includes a subpleural and basal predominance of lesions, reticular abnormalities, honeycombing with or without traction

Respiration 2017;93:363-378 367 

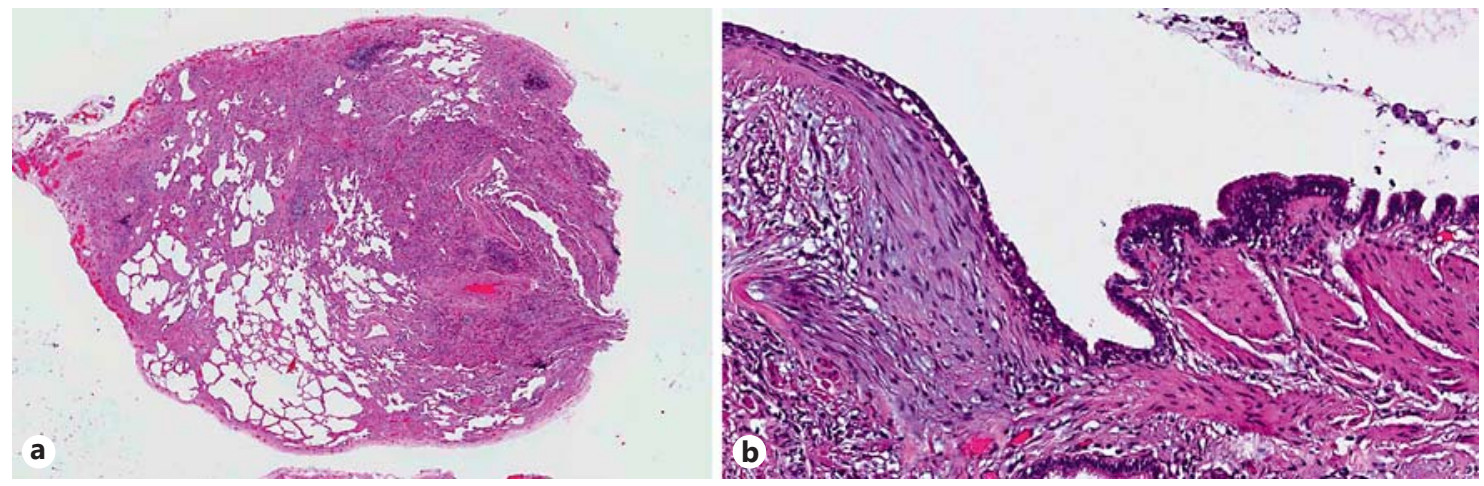

Fig. 2. a Typical histopathological UIP (usual interstitial pneumonia) pattern with a heterogeneous distribution and fibroblast foci. b Typical fibroblast foci with myofibroblast accumulation.

bronchiectasis, and an absence of features inconsistent with a UIP pattern (Table 1; Fig. 1). Identifiable causes need to be excluded. If radiological imaging is inconsistent with a UIP, biopsy is required to confirm the UIP pattern (Fig. 2) and a diagnosis should be established after multidisciplinary discussion (MDD) [1]. A modified diagnostic algorithm from the international guidelines [1] has previously been proposed [8]. Detailed descriptions of imaging (Table 1) and histopathological UIP patterns are also summarized in the international ATS/ERS guidelines [1].

Which Physiological Tests Should Be Performed at the Time of Diagnosis (Evaluation of Prognosis) and for

Follow-Up in Patients with Suspected IPF?

Although physiological testing is not formally required for an initial diagnosis of IPF according to the ATS/ERS/ JRS/ALAT, some parameters, such as a DLCO $<40 \%$, are predictive of increased mortality, and changes in FVC signal disease progression [1]. In the German guidelines for diagnosis and treatment of IPF, lung functional changes with a decline in FVC $\geq 10 \%$ or a decline in FVC $<10 \%$ combined with a decrease in DLCO, deteriorated arterial blood gas analysis or 6-min walk test (6-MWT) results, and clinical symptoms (dyspnea and cough) should be used to determine disease progression and prognosis [3]. In addition, the German consensus conference considers changes in FVC or inspiratory vital capacity as equal parameters [3]. The current French recommendation suggest a combination of symptoms (severity of dyspnea), lung functional tests (FVC and DLCO), the 6-MWT, the extent of honeycombing on HRCT, pulmonary hypertension $(\mathrm{PH})$ on echocardiography, and using a score to assess prognosis [4].
In addition to DLCO and spirometry (FVC) to assess disease prognosis and progression in accordance with international recommendations, we suggest performing body plethysmography in search of a restrictive ventilatory disorder. The 6-MWT (in search of exercise-induced oxygen desaturation) should be performed routinely at the time of diagnosis in patients with suspicion of IPF as a reference value to evaluate disease progression. Arterial blood gas analysis should not be performed routinely, unless long-term oxygen therapy is considered. Spiroergometry may be performed if driven by a specific clinical question. (Very good consensus) (1-6 $=0$ votes, $7=10 \%$ of votes, $8=10 \%$ of votes, $9=80 \%$ of votes)

\section{Which Laboratory Tests Should Be Performed at the}

Time of Diagnosis of Suspected IPF?

Serological analyses should be performed according to international guidelines [1] to exclude secondary causes, specifically since some rheumatologic diseases may manifest as lung fibrosis before systemic symptoms become apparent $[1,3]$. International recommendations according to the ATS/ERS/JRS/ALAT include systematic serological testing even in the absence of symptoms of connective tissue disease, such as rheumatoid factor, anti-cyclic citrullinated peptide, and anti-nuclear antibody titer and pattern. Further serological testing (anti-Jo-1, -SSA, -SSB, and -Scl-70) is only considered in selected cases because of an unclear benefit [1]. The German recommendation adopts this proposal [3]. In order to exclude connective tissue disease-associated ILDs, the French recommendations suggest assessing (1) differential blood cell counts, as well as C-reactive protein, serum creatinine, transaminase, $\gamma$-glutamyltransferase, and alkaline phosphatase; (2) anti-nuclear antibodies, anti-citrullinated cyclic peptide antibodies, and rheumatoid factor; and (3), depending on the clinical picture or if anti-nuclear anti- 
bodies are present, antibodies specific to the Sjögren syndrome (anti-SSA and anti-SSB), systemic sclerosis antibodies (anti-centromere, anti-topoisomerase-1, and antiU3RNP antibodies), anti-synthetase antibodies, antithyroid antibodies, creatinine phosphokinase, and serum electrophoresis [4]. A recent review suggests anti-nuclear antibodies, Jo-1, SSA, SSB, rheumatoid factor, anti-CCP, and anti-neutrophil cytoplasmic antibodies as screening markers for all patients with unclear ILD [32].

We recommend routine blood testing including erythrocyte sedimentation rate, differential blood cell count, C-reactive protein, and renal (blood, urine dip stick) and liver function, as well as serological markers for systemic autoimmune diseases (antinuclear antibodies, rheumatoid factor, and anti-cyclic citrullinated peptide). These tests may be repeated during the disease course if new clinical aspects appear. We suggest performing expanded serological testing when an autoimmune disease is suspected based on a thorough patient history and clinical examination. This may include markers for mixed connective tissue disease (anti-U1 ribonucleoprotein antibodies), Sjögren syndrome (anti-SSA and anti-SSB antibodies), and systemic lupus erythematosus (Sm, dsDNA), as well as specific antibodies for idiopathic inflammatory myopathies (anti-Jo-1, anti-PL7, and antiPL12), systemic sclerosis (anti-centromere, anti-topoisomerase-1, and anti-U3RNP), and vasculitis (anti-neutrophil cytoplasmic autoantibodies). Serological markers for inflammatory myopathies (e.g., anti-Jo-1 antibodies) may also be determined in asymptomatic patients, since ILD can occur with or without preceding myopathy. (Very good consensus) (1-6 = 0 votes, $7=10 \%$ of votes, $8=30 \%$ of votes, $9=60 \%$ of votes)

\section{Should Patients with a UIP Pattern and Clinical or} Serological Autoimmune Features Who Do Not Meet the Definite Diagnostic Criteria for a Connective

Tissue Disease Be Considered as Having IPF?

Some patients present with clinical features suggesting an underlying autoimmune process, but they do not meet the established criteria for a connective tissue disease; this has been called "interstitial pneumonia with autoimmune features" (IPAF). Evidence of treatment for this newly described population is missing [33].

We recommend that the diagnosis and treatment decisions for interstitial pneumonia with autoimmune features be left with experienced MDD groups. (Very good consensus) (1-7 = 0 votes $8=10 \%$ of votes, $9=90 \%$ of votes)

\section{Should Bronchoscopy and BAL Be Performed if IPF Is} Suspected?

Lymphocytosis $\leq 15 \%$ or eosinophilia $\leq 1 \%$ in BAL fluid are considered normal. Alterations in cytopathological cell distribution in BAL fluid should initiate a search for a diagnosis other than IPF $[34,35]$. In the guidelines from the ATS/ERS/JRS/ALAT, BAL is not routinely recommended [1]. In contrast, routine BAL to investigate ILD is recommended by the German IPF guidelines [3]. The French recommendation also includes BAL in suspected IPF, especially if HRCT does not show a definite UIP pattern [4]. Controlled studies are needed to establish the true benefit of BAL for ruling out differential diagnoses.

We recommend performing BAL to search for other causes of a fibrotic disease, especially chronic hypersensitivity pneumonitis and fibrotic nonspecific interstitial pneumonia, in patients with suspected IPF if the risk of performing BAL is considered inferior to the possible benefit to diagnostic accuracy. If chest imaging does not show a definite UIP pattern, BAL and a biopsyconfirmed UIP diagnosis should be aimed at. Not performing BAL is justified, specifically if the individual respiratory situation considerably increases the risk of the procedure. (Very good consensus) $(1-6=0$ votes, $7=10 \%$ of votes, $8=0$ votes, $9=90 \%$ of votes)

\section{If Indicated, Which Type of Histological Biopsy Should} Be Performed: Transbronchial Biopsy, Cryobiopsy, or SLB?

International guidelines discourage the use of transbronchial biopsy in IPF patients, with a weak recommendation due to low-quality evidence $[1,3]$. Meanwhile, new techniques have been developed. Cryobiopsy has shown good diagnostic yield in initial studies and can be performed safely in comparison to SLB [36]. Cryobiopsy has increased diagnostic confidence in multidisciplinary diagnosis of IPF [37]. Nevertheless, its diagnostic accuracy has not been properly evaluated in direct comparison to SLB [38]. Cryobiopsy might be an additional diagnostic option in the future if performed in experienced centers; however, is not routinely used in Switzerland at the present time. In unclear cases, surgical biopsy (videoassisted thoracoscopy) may still be needed to achieve a diagnosis. Possible risks of performing video-assisted thoracoscopy and cryobiopsy need to be considered.

We do not recommend transbronchial biopsy for suspected IPF. We recommend performing video-assisted thoracoscopic SLB if ILD remains unclassifiable or possible UIP is diagnosed after an MDD and if the patient-specific risk of performing SLB is considered inferior to its benefit to diagnostic accuracy. SLB should be performed only if the result is expected to strongly influence therapeutic decisions, especially if the choice between antifibrotic and anti-inflammatory/immunosuppressive treatment is difficult in idiopathic interstitial pneumonia with an unclear pattern. SLB should not be performed if it will not result in any treatment decision. SLB should not be performed in patients with severely impaired lung functional testing results, significant comorbidities, or advanced biological age. The sites of lung biopsy should be chosen in an MDD with a chest physician, radiologist, and thoracic surgeon. Whenever possible, samples should be tak-

Idiopathic Pulmonary Fibrosis in

Switzerland

Respiration 2017;93:363-378 
en from at least 2 lobes. The tips of the middle lobe and of the lingula should be avoided, as well as areas with exclusive honeycombing. Cryobiopsy may be considered as an alternative approach in experienced centers if there are no contraindications. Not performing biopsy may be an acceptable option for individual patients, specifically if the radiological signs favor possible UIP. (Very good consensus) (1-5 $=0$ votes, $6=10 \%$ of votes, 7-8 $=0$ votes, $9=90 \%$ of votes)

\section{What Is the Role of MDD?}

MDD is considered the gold standard for IPF diagnosis and should involve at least a pulmonologist, a radiologist, and a pathologist with expertise in ILD [1, 3, 39]. MDD has been shown to increase diagnostic confidence regarding IPF in this setting [40]. With IPF being a rare disease, patients should be referred to expert centers for diagnosis, and their cases should be discussed in an experienced ILD MDD to achieve the highest possible diagnostic accuracy - even more so because interobserver agreement between radiologists regarding UIP on HRCT is only moderate independently of their respective clinical experience [41].

We recommend MDD with at least a pulmonologist, a radiologist, and a pathologist (all with ILD expertise) at experienced centers for all IPF diagnoses, both before and after biopsy. MDD before surgical biopsy should suggest the site of biopsy to improve diagnostic outcome. Treatment suggestions should be formulated in MDD whenever possible. (Perfect consensus) (1-8= 0 votes, $9=100 \%$ of votes)

Should Genetic Mutations Be Searched for once the Diagnosis Is Established?

The 2011 international guidelines did not recommend genetic screening for IPF patients [1]. Meanwhile, several associations between IPF and genetic mutations or polymorphisms have been identified. Some genetic variants are associated with increased or reduced survival and may predict disease outcome [28, 29]. Knowledge of these genetic determinants might affect the timing of referral to lung transplantation centers. Genetic counseling can also help to identify familiar forms of fibrosis. In addition, some polymorphisms may influence the response to specific treatments, such as TOLLIP mutations for N-acetylcysteine (NAC) treatment [42]. Although the German guidelines did not recommend routine screening yet, they underlined the current change of knowledge in this field [3].

We recommend genetic testing for gene mutations if familial fibrosis is suspected or IPF occurs at a younger age ( $<50$ years). At the present time, we do not suggest searching for genetic polymorphisms (MUC5B, etc.) in the routine clinical setting for all IPF patients. (Very good consensus) (1-4 $=0$ votes, $5=10 \%$ of votes, $6-7=0$ votes, $8=10 \%$ of votes, $9=70 \%$ of votes)

\section{Comorbidities}

Comorbidities and complications are common in IPF patients [43]. These patients have an increased risk of lung cancer [43]. Since IPF itself reduces life expectancy, it is unclear today if screening methods recommended for risk populations are of any benefit to these patients [44]. $\mathrm{PH}$ [45], obstructive sleep apnea syndrome [46], gastroesophageal reflux [15], and coronary heart disease are more common in IPF patients than in the general population [1]. Examinations to diagnose or exclude comorbidities depend on symptoms and clinical presentation.

\section{Current Recommendations for Treatment}

The approach to treatment for IPF has considerably changed over the past few years. Many previously used treatments have been shown to be ineffective and are therefore no longer recommended. Specifically, immunosuppressive therapies - e.g., triple therapy with prednisone, azathioprine, and NAC - did not show any benefit and were even considered harmful [47]. Other ineffective treatments are listed in the current international guidelines $[1,2]$.

A novel category of treatment has emerged out of recent clinical trials. Antifibrotic drugs include currently 2 substances, pirfenidone and nintedanib, which mainly target fibrosis progression. Pirfenidone is an antifibrotic agent that interferes with the TGF- $\beta$ pathway, although its exact mode of action remains unclear. In vitro studies have shown that pirfenidone inhibits fibroblast proliferation and differentiation into myofibroblasts through inhibition of TGF- $\beta$ [48]. In 2 out of 3 placebo-controlled clinical randomized phase III trials (CAPACITY: PIPF004, PIPF-006, and ASCEND), pirfenidone significantly slowed the decline in FVC $[49,50]$. The decline in FVC was reduced by $193 \mathrm{~mL}$ in pirfenidone-treated patients compared to placebo-treated ones after 52 weeks in the ASCEND trial including 555 IPF patients in total [50]. The diagnosis of IPF was confirmed if the diagnostic criteria according to the current ATS/ERS guidelines were met (i.e., exclusion of an alternative diagnosis of ILD with a definite UIP pattern on HRCT or possible UIP with surgical biopsy showing a UIP pattern) [1]. If radiological imaging showed greater emphysematous changes than fibrotic changes, patients were excluded [50]. The ASCEND trial showed prolonged progression-free survival among pirfenidone-treated patients after 52 weeks [50]. A pooled data analysis of the CAPACITY and ASCEND trials suggests a positive effect on mortality $(p=$

Funke-Chambour et al. 
0.011) [50]. Reported side effects are mainly gastrointestinal and skin-related events during clinical trials and long-term observations [51]. It is recommended that pirfenidone be taken with meals to reduce gastrointestinal side effects. Spacing the ingestion of the 3 tablets and prokinetic medication as well as proton pump inhibitors can reduce side effects [52]. Because of increased photosensitivity, patients should be advised to avoid direct exposure to sunlight, to use sunscreens, and to wear protective clothing outside [52]. Pirfenidone should not be used with end-stage renal disease and severe hepatic dysfunction (Child-Pugh class C). Liver function needs to be monitored prior to treatment, as well as regularly during treatment (initially every month for 6 months, then every 3 months if stable) [52]. The concomitant use of fluvoxamine should be avoided because of decreased drug clearance through inhibition of CYP1A2. Ciprofloxacin reduces pirfenidone clearance by the same mechanism. Omeprazole might also influence the pharmacokinetics of pirfenidone [53].

Pirfenidone has been recommended for patients with IPF (weak recommendation, moderate evidence) by the German [3] as well as international guidelines (conditional or weak recommendation for use, moderate confidence in estimates of effect) [2]. The French guidelines recommend its use in mild-to-moderate IPF [4].

Nintedanib is a tyrosine kinase inhibitor targeting PDGF, FGF, and VEGF receptors. The antifibrotic mechanisms of tyrosine kinase inhibition by nintedanib have been extensively studied [54], and inhibitory effects on proliferation and extracellular matrix production have been shown in lung fibroblasts derived from IPF patients [55]. In clinical trials, 2 concurrent phase III studies (INPULSIS-1 and -2) were conducted with a centrally reviewed diagnosis of IPF [56]. IPF was confirmed if specific criteria were met [56]. Both studies showed a significant reduction of the decline in FVC (a difference of 124 and $94 \mathrm{~mL}$, respectively, between treated patients and placebo controls, $p<0.001)$ in patients with the following inclusion criteria: $\mathrm{FVC}>50 \% \mathrm{FEV}_{1}$ (forced expiratory volume in $1 \mathrm{~s}) / \mathrm{FVC}>0.7$; and DLCO $30-79 \%$. In a prespecified, pooled analysis of a key secondary endpoint, the time to first acute exacerbation was reduced [56]. However, in individual studies, only INPULSIS-2 showed a significant benefit on time to first exacerbation. Another secondary endpoint (change in total score on the St. George Respiratory Questionnaire [SGRQ]) did not reveal any statistically significant difference in pooled analyses [56]. No beneficial effect on survival has been reported, including in pooled analyses, although a trend

Idiopathic Pulmonary Fibrosis in Switzerland towards reduced all-cause mortality and respiratory mortality was observed, with reduced on-treatment mortality and time to first investigator-reported acute exacerbation when phase II and phase III studies were combined [57]. The main side effects of nintedanib reported were diarrhea, nausea, abdominal pain, and vomiting [56]. Although diarrhea is a common side effect, it is mild to moderate in most cases and can be treated with antimotility drugs [52]. Liver function needs to be checked prior to treatment and every month for 3 months, then every 3 months [52]. Nintedanib should not be used in case of liver disease (Child-Pugh classes B and C) [52]. Its safety and efficacy in severe renal failure have not been evaluated, and treatment is thus not recommended [52]. Treatment should be conducted with care if known cardiovascular diseases or significant risk factors are present and the risk of bleeding is increased [52], although no clear contraindication has been formulated.

Recently, 2 network meta-analyses investigated studies on IPF and found consistently beneficial results for both pirfenidone and nintedanib in comparison to other treatments. No superiority of either drug could be demonstrated. The conclusion about mortality was divergent and needs to be considered carefully $[58,59]$.

Similar to pirfenidone, nintedanib is now recommended by international guidelines (conditional or weak recommendation for use, moderate confidence in estimates of effects) [2]. Study data were not available yet when the German and French recommendations were published.

We suggest treatment with pirfenidone or nintedanib for patients with IPF. In the absence of direct comparisons, none of the 2 drugs can currently be considered superior to the other. The effect on decline in FVC appears of a similar magnitude with either of the 2 drugs. At the present time, the choice between these 2 drugs should therefore mainly rely on preferences regarding the side effect profile, contraindications to treatment, and comorbidities. (Very good consensus) (1-6 $=0$ votes, $7=10 \%$ of votes, $8=10 \%$ of votes, $9=70 \%$ of votes)

\section{When Should Antifibrotic Treatment Be Started?}

Randomized trials of pirfenidone (ASCEND) and nintedanib (INPULSIS) on IPF included patients with moderately severe disease (FVC 50-90 and $>50 \%$, DLCO $30-90$ and $30-79 \%$, and $\mathrm{FEV}_{1} / \mathrm{FVC}>80$ and $>70 \%$, respectively; 6-MWT distance $>150 \mathrm{~m}$ in ASCEND only) $[50,56]$. However, the benefit from therapy might not be related to disease stage. In patients treated with nintedanib, whether the initial FVC was $<70$ or $>70 \%$ did not significantly influence the drug's effect on decline in FVC,

Respiration 2017;93:363-378 
SGRQ score, or time to first exacerbation, although in patients with a lower FVC, more acute exacerbations and greater deterioration were reported in the placebo-treated group [60]. In real-life observations, pirfenidone showed efficacy in reducing the decline in FVC in more advanced disease [61]. Rapid lung functional decline or exacerbation can occur at any stage. Thus, long observational periods without treatment may not be useful. A patient's preferences regarding treatment need to be included in the decision-making process about initiation of therapy. Patients should be clearly informed about the expected disease course and the risks and benefits of therapy - especially the fact that treatment will not improve lung function but only slow down disease progression, and that they likely will not feel any improvement of symptoms or quality of life. On the other hand, early treatment may preserve lung function and avoid additional respiratory symptoms [53].

We suggest that antifibrotic treatment be proposed upon diagnosis of IPF, especially in patients with documented disease progression as shown by worsening of symptoms, lung functional impairment, or chest imaging. An observational period without antifibrotic treatment may be appropriate in selected patients with mild and stable disease and comorbidities. If no treatment is started, patients should be followed up every 3-6 months and be reevaluated for treatment initiation. The choice of the initial drug should depend on a patient's preferences, comorbidities, and concomitant treatments. (Very good consensus) (1-7 = 0 votes, $8=10 \%$ of votes, $9=90 \%$ of votes)

Should We Treat Patients with Probable or Possible $I P F$, and with Fibrotic Lung Disease other than IPF?

Patients with possible IPF were included in the INPULSIS studies with nintedanib. A prespecified subgroup analysis (sex, age, race, baseline FVC, systemic corticosteroid use, etc.) showed consistent effects of nintedanib on possible UIP compared to definite UIP [62]. The diagnosis of probable and possible IPF should involve MDD.

Lung fibrosis other than IPF might have overlapping pathogenic disease mechanisms, although there are clear differences in disease course and prognosis in patients with lung fibrosis other than IPF. In contrast to IPF, fibrotic ILD occurring in connective tissue disease patients may benefit from immunosuppressive therapy. Although the studies are ongoing, the effect of pirfenidone or nintedanib on ILD associated with connective tissue disease is currently unknown [63].

We suggest that only patients with definite IPF be treated with antifibrotic agents. Treatment of probable and possible IPF should be discussed in MDD. We suggest not using antifibrotic drugs for diseases other than IPF until evidence on drug benefits is available. (Very good consensus) (1-2 $=0$ votes, $3=10 \%$ of votes, $4=0$ votes, $5=10 \%$ of votes, $6-7=0$ votes, $8=20 \%$ of votes, $9=60 \%$ of votes)

Should Patients with Severely Impaired Lung Function Be Treated with Pirfenidone or Nintedanib?

New data are emerging that support the use of antifibrotic drugs outside the lung functional values that were used for inclusion in studies on pirfenidone and nintedanib. Pirfenidone seems to be more effective in rapidly progressive IPF [64]. For nintedanib, FVC at baseline seems not to influence treatment effects [65].

We recommend that treatment with antifibrotic medication should also be offered to patients with severe forms of IPF if palliation is not yet considered. (Very good consensus) (1-6 = 0 votes, $7=10 \%$ of votes, $8=0$ votes, $9=90 \%$ of votes)

Should We Treat Patients with Combined Pulmonary Fibrosis and Emphysema Syndrome?

Combined pulmonary fibrosis and emphysema (CPFE) is a clinicoradiological syndrome characterized by the simultaneous occurrence of both emphysema (usually of the paraseptal type with predominance in the upper lung zones) and ILD (usually in the lower lung zones) in variable proportions on chest CT [66-68]. These patients have a particular lung functional profile with near-normal lung volumes and severely reduced DLCO, resulting on the one hand from opposed effects of fibrosis and emphysema on lung mechanics, and on the other hand from additive effects on gas exchange. $\mathrm{PH}$ is a common feature of CPFE and is associated with poor prognosis [69]. The decline in lung volume over time was reported to be milder in CPFE than in IPF, but survival appears similarly poor $[70,71]$. It is unclear whether CPFE is a single disease or the simultaneous occurrence of 2 disorders sharing 1 or more risk factors such as smoking. Emphysema in IPF patients is associated with worse prognosis [72]. The fibrotic component of CPFE could correspond to UIP on imaging or histologically. However, other histological patterns have been reported, such as nonspecific interstitial pneumonia [73]. Patients with emphysema in addition to IPF have been excluded from the pirfenidone trials, but not from the nintedanib trial.

We recommend treating the fibrosis component of CPFE with pirfenidone or nintedanib if this component meets the diagnostic criteria for IPF and the fibrotic disease is predominant over emphysematous areas. (Very good consensus) (1-6 = 0 votes, $7=10 \%$ of votes, $8=10 \%$ of votes, $9=80 \%$ of votes)
372

Respiration 2017;93:363-378

DOI: $10.1159 / 000464332$
Funke-Chambour et al. 
When Should Antifibrotic Treatment Be Stopped or

Switched?

Clinical trials of IPF therapy with pirfenidone followed subjects up to 72 months [49]. Pirfenidone has been well tolerated and documented for nearly 10 years of treatment $[51,74]$. Sustained differences in lung functional decline were observed between intervention and placebo groups in those trials over time. In contrast, cessation of antifibrotic therapy has not specifically been studied. Given that pathophysiological IPF mechanisms evolve over months or years and are slowed but neither stopped nor reversed by either nintedanib or pirfenidone, specific therapy should generally not be stopped. One recent study showed that in patients with IPF progression despite pirfenidone therapy, treatment maintenance was associated with better outcome when compared to patients progressing under placebo [75]. In IPF patients with significant side effects, stopping or reducing antifibrotic therapy may be necessary. It has also been reported that switching from one drug to another was safe [76].

In IPF patients treated with antifibrotic drugs, disease progression should not be interpreted as treatment failure and lead to treatment withdrawal, provided that treatment tolerance is satisfactory. If intolerable side effects occur, a treatment switch might be considered. (Very good consensus) (1-6 $=0$ votes, $7=$ $10 \%$ of votes, $8=0$ votes, $9=90 \%$ of votes)

\section{Should Pirfenidone and Nintedanib Be Combined?}

The safety and pharmacokinetics of the combination of pirfenidone and nintedanib have been studied. Nintedanib showed lower serum levels when added to pirfenidone in this study [77]. Studies examining the efficacy of combination therapies have not been made until today.

In the current absence of evidence regarding the benefit of combined therapy, we recommend that combining pirfenidone and nintedanib in IPF patients be avoided. (Very good consensus) $(1-6=0$ votes, $7=10 \%$ of votes, $8=0$ votes, $9=90 \%$ of votes)

\section{Should Patients Be Treated with NAC?}

High-dose NAC treatment (600 mg $3 \times$ /day) had been considered standard therapy in addition to azathioprine and prednisone until the PANTHER trial showed increased mortality and morbidity in its triple-therapy arm, which was therefore prematurely stopped [47]. Administration of high-dose NAC versus placebo did not show any benefit [78]. A recent randomized controlled trial examining the effect of NAC or placebo on IPF patients treated with pirfenidone did not find any benefit from the addition of NAC [79]. One study investigated the effects of

Idiopathic Pulmonary Fibrosis in Switzerland
NAC on death, transplantation, hospitalization, or decline of $\geq 10 \%$ in FVC depending on different single nucleotide polymorphisms (SNPs) within TOLLIP or MUC5B. While one SNP within TOLLIP (rs3750920 TT genotype) significantly reduced these specific endpoints, another SNP (TOLLIP CC genotype) showed a trend towards harm [42]. Thus, without systematic genotyping and additional studies, no therapeutic recommendation is possible.

We do not recommend high-dose NAC treatment for IPF. (Perfect consensus) (1-8 $=0$ votes, $9=100 \%$ of votes)

\section{Lung Transplantation}

\section{When Should IPF Patients Be Considered for \\ Transplantation?}

Lung transplantation is a well-accepted procedure for IPF and should be considered for patients without significant comorbidities or obvious contraindications. $\mathrm{Ab}$ solute and relative contraindications to transplantation are similar to other indications for lung transplantation [80], but relative contraindications in particular vary among centers in Switzerland.

The possibility of performing lung transplantation should be considered for all patients with IPF at the time of diagnosis, even if their lung function initially is well preserved, because of the poor prognosis of the disease [80]. In general, IPF patients under 65 years of age without significant comorbidities or contraindications should be referred early to a transplantation center for first assessment and information about the benefits and risks of lung transplantation. Referral should specifically be considered when lung function is impaired and/or rapidly declining.

Timely referral is crucial as the disease may progress rapidly. The time point at which to place a patient on the waiting list depends on the expected waiting time on the list, which is determined by the organ allocation system. In general, wait-listing is indicated when a patient's lung function is severely impaired or rapidly deteriorating, manifesting itself in the following features (none of which should be used in isolation): (a) lung function declined (FVC by $\geq 10 \%$ or DLCO by $\geq 15 \%$ over 6 months); (b) $\mathrm{O}_{2}$ desaturation declined to $<88 \%$; (c) total walking distance declined to $<250 \mathrm{~m}$ in the 6-MWT; (d) walking distance declined by $>50$ m over 6 months; (e) a diagnosis of $\mathrm{PH}$ is established; or ( $\mathrm{f}$ ) the patient needs hospitalization because of respiratory decline, pneumothorax, or acute exacerbation [80]. Once a patient is listed for lung trans-

Respiration 2017;93:363-378 373 
plantation, the transplantation center in close collaboration with the treating pulmonologist should optimize every aspect of the candidate's condition in order to maintain the patient suitable for transplantation. Patients should participate in a pulmonary rehabilitation program.

In case of acute exacerbations while on the waiting list, patients may be eligible for invasive ventilation or extracorporeal membrane oxygenation in case of single-organ failure, also as a "bridging measure" until transplantation. However, patients have to be selected very carefully in order to achieve acceptable posttransplantation outcomes. Eligibility criteria for "bridging" strategies in this scenario differ between centers [81].

The possibility of performing lung transplantation should be considered for all patients with IPF at the time of diagnosis, even if their lung function initially is well preserved. In particular, IPF patients under 65 years of age without significant comorbidities or contraindications and clinical and functional deterioration despite antifibrotic therapy should be referred early to a transplantation center for initial assessment. Wait-listing is indicated when a patient's lung function is severely impaired or rapidly deteriorating based on a combination of clinical and physiological parameters following careful evaluation. Once a patient is listed for lung transplantation, the transplantation center and the treating pulmonologist should cooperate to maintain the patient suitable for transplantation. (Good consensus) ( $1=\mathbf{2 0} \%$ of votes, $2=0$ votes, $3=10 \%$ of votes, $4-7=0$ votes, $8=10 \%$ of votes, $9=60 \%$ of votes)

\section{Should Treatment Be Stopped if Patients Are Listed} for Lung Transplantation?

Inhibition of the TGF- $\beta$ pathway as well as inhibition of tyrosine kinases might theoretically interfere with the wound healing process. So far, no increased risks of operative or postoperative complications have been reported for IPF patients on either of these antifibrotic drugs.

In general, stopping antifibrotic therapy is not recommended for patients on a waiting list for lung transplantation. However, a medical therapy should be performed after wait-listing in close collaboration between the transplantation center and the treating pulmonologist. (Perfect consensus) (1-8 $=0$ votes, $9=100 \%$ of votes)

\section{General Recommendations}

While information on the vaccination of patients with chronic lung diseases is summarized in the ACIP recommendations on the CDC Web page [82], we decided to vote on the following topics.

\section{Pulmonary Rehabilitation and Long-Term Oxygen}

Therapy

IPF patients benefit from pulmonary rehabilitation, which has been shown to improve quality of life and exercise capacity [83]. Exercise capacity and fatigue can predict general physical activity in patients with IPF [84].

Although few studies are available, a retrospective analysis suggested benefits from oxygen supplementation for exercise performance in IPF patients [85].

We recommend prescribing pulmonary rehabilitation to patients with IPF. We also recommend prescribing oxygen therapy during exercise if hypoxemia limits the exercise capacity, and home oxygen therapy if $\mathrm{PaO}_{2}$ reaches the standard threshold of $55 \mathrm{~mm} \mathrm{Hg}(7.33 \mathrm{kPa})$, or of $60 \mathrm{~mm} \mathrm{Hg}(8 \mathrm{kPa})$ in case of polyglobulia or right-sided heart insufficiency. (Perfect consensus) (1$8=0$ votes, $9=100 \%$ of votes)

\section{Mechanical Ventilation}

Acute respiratory failure may be treated by high-flow nasal oxygen, noninvasive ventilation, or invasive mechanical ventilation. Few data are available on the role of high-flow nasal oxygen in IPF [86]. Invasive mechanical ventilation has been consistently associated with a very high mortality among IPF patients in the ICU setting (>90\%) [87]. However, in a recent, large, nationwide cohort analysis in the USA, in-hospital mortality among patients with IPF requiring invasive mechanical ventilation was approximately $50 \%$ [88]. This trend may also be due to contemporary management approaches to acute lung injury (lung-protective ventilation strategies). Noninvasive ventilation can be used in case of acute exacerbation and respiratory failure (hypercapnia) in selected patients with hypoxemic respiratory failure in order to try to prevent endotracheal intubation. Acceptance and setting of noninvasive ventilation might be difficult to achieve in IPF patients with altered lung mechanics, and the outcome of patients failing noninvasive ventilation is poor $[89,90]$.

For patients with IPF and acute respiratory failure, we suggest to use invasive mechanical ventilation if a reversible cause such as an infection, pulmonary embolism, heart failure, or other is present. In acute exacerbation of IPF, which is associated with a poor prognosis, the benefit from invasive ventilation is less clear and should be discussed on a case-by-case basis. Noninvasive ventilation can be used. If invasive mechanical ventilation is required for elective surgery, the ventilation strategy should be planned in advance and should aim at reducing the risk of triggering an acute exacerbation. (Very good consensus) (1-6 $=0$ votes, $7=10 \%$ of votes, $8=0$ votes, $9=90 \%$ of votes)
Respiration 2017;93:363-378 DOI: $10.1159 / 000464332$
Funke-Chambour et al. 


\section{Palliation}

In general, advanced care planning and palliative care are still neglected for IPF patients. Many IPF patients die in a hospital setting with late referral for palliation [91]. Underuse of palliative care has been reported for patients with IPF [92]. Palliative care should be discussed early in the course of IPF with patients who are ineligible for lung transplantation or with a poor lung function or performance status. Patients' values, goals, and therapeutic preferences should be taken into account. A decision to stop IPF-specific therapy should be based on individual patient assessment.

We recommend introducing palliative care on an individual case basis. Patients with severe IPF may choose not to be treated with antifibrotic drugs. This should be discussed with these patients and be respected. Nevertheless, pharmaceutical and nonpharmaceutical treatment options should be proposed to all patients. (Very good consensus) (1-3 $=0$ votes, $4=20 \%$ of votes, $5-8=0$ votes, $9=80 \%$ of votes)

\section{Selected Comorbidities and Complications: Acute Exacerbation and PH}

\section{How Should Acute Exacerbation of IPF Be Treated?}

Secondary causes of respiratory failure should be searched for, since they might influence prognosis and therapy [25]. In patients with severe respiratory impairment, exclusion of infection by invasive procedures such as BAL is often difficult; therefore, empirical antibiotic treatment is often used [93]. In addition, antiviral treatment can be indicated according to the clinical circumstances [94]. Intravenous corticosteroids as early as possible are the recommended treatment, but evidence on their efficacy is lacking [1]. The benefit from initiating pirfenidone or nintedanib treatment during acute exacerbation is unknown.

We suggest not starting pirfenidone or nintedanib treatment at the time of acute exacerbation for patients with IPF requiring hospital admission, but previous treatment might be continued. As a practical approach, we suggest that antibiotic treatment should be administered if an infection cannot be excluded with certainty. The role of corticosteroids remains unclear. A short course of steroids (i.e., administration of intravenous methylprednisolone for a few days) may be considered in some cases. However, evidence on the efficacy of this treatment is lacking. (Very good consensus) (1-3 $=0$ votes, $4=10 \%$ of votes, $5-6=$ 0 votes, $7=10 \%$ of votes, $9=80 \%$ of votes)

\section{Should PH Be Treated in IPF Patients?}

$\mathrm{PH}$ occurs in IPF patients [43]. In patients with mildto-moderate restriction due to IPF, $\mathrm{PH}$ was found in $24 \%$ of the cases [95]. In patients with mild ILD but severe $\mathrm{PH}$, it may be difficult to determine whether $\mathrm{PH}$ is secondary to lung disease or if the patients have pulmonary arterial hypertension in addition to a chronic lung disease. Current guidelines do not recommend treatment of $\mathrm{PH}$ in IPF [45]. In addition, a recent study on riociguat in PH due to idiopathic ILD had to be prematurely stopped because of increased mortality [96]. Because of the poor prognosis, specific $\mathrm{PH}$ therapy for IPF patients with a mean pulmonary artery pressure $\geq 35 \mathrm{~mm} \mathrm{Hg}$ should be evaluated at a center with expertise in $\mathrm{PH}$ where there is also expertise in ILD for individualized patient care [45]. A recent review on behalf of the Swiss Society for Pulmonary Hypertension summarizes the challenges posed by $\mathrm{PH}$ in chronic lung diseases including ILD [97].

We recommend that specific treatment of $\mathrm{PH}$ in IPF should not be introduced if $\mathrm{PH}$ can be reasonably attributed to IPF, i.e., if it corresponds to group 3.2 of the international $\mathrm{PH}$ classification. If $\mathrm{PH}$ is suspected to occur independently of IPF, a specific evaluation should be done at a center with expertise in $\mathrm{PH}$ and ILD. (Very good consensus) (1-8 $=0$ votes, $8=10 \%$ of votes, $9=90 \%$ of votes)

\section{Should Gastroesophageal Reflux Be Treated in IPF?}

Acid gastroesophageal reflux is considered to contribute to epithelial cell injury and thus to the pathogenesis of IPF. Whether Helicobacter pylori is involved in the pathogenesis of IPF remains unclear, since a recent study could not show its presence in lung biopsies [98]. Nevertheless, gastroesophageal reflux is considerably prevalent in patients with IPF. Anti-reflux treatment, however, has been controversially discussed [99]. One prospective observational study showed reduced disease progression regarding FVC and exacerbations [100], while a new post hoc evaluation of clinical studies on IPF shows increased numbers of overall and pulmonary infections in advanced IPF patients treated with antacids compared to those not treated with antacids [101]. Furthermore, patients with antacid treatment at baseline receiving nintedanib seemed to do worse than those without antacid treatment [99]. Promising surgical interventions to inhibit acid as well as anti-acid reflux with Nissen fundoplication in IPF are currently studied in an ongoing clinical trial (WRAPIPF) [99]. Current international guidelines recommend conditional treatment with proton pump inhibitors regardless of patients' gastroesophageal reflux status [2], which is controversially discussed [99].

We recommend treating symptomatic reflux in IPF patients, but not asymptomatic reflux, since evidence is missing. (Very good consensus) (1-5 $=0$ votes, $6=10 \%$ of votes, $7=10 \%$ of votes, $8=10 \%$ of votes, $9=70 \%$ of votes)
Idiopathic Pulmonary Fibrosis in Switzerland
Respiration 2017;93:363-378 DOI: $10.1159 / 000464332$
375 
How Should Cough and Dyspnea Be Treated

Symptomatically in IPF?

Chronic cough and dyspnea are the main symptoms presented by IPF patients [1]. Unfortunately, current antifibrotic treatments have not been shown to improve these symptoms. Current antitussive medications have only limited benefit, and thalidomide has been shown to reduce cough but has not been routinely used due to possible toxicities [53]. In Switzerland, some patients improved under azithromycin treatment, possibly due to immunomodulatory mechanisms. This treatment is investigated in a placebo-controlled trial in Switzerland that is currently recruiting [102].

We suggest that chronic cough and dyspnea are empirically treated with currently available antitussive drugs, as well as with opioids for advanced dyspnea in palliative situations. For chronic cough, inducing factors need to be searched for and treated accordingly. These include reflux and obstructive sleep apnea syndrome, which are common comorbidities in IPF. (Very good consensus) $(1-6=0$ votes, $7=10 \%$ of votes, $8=20 \%$ of votes, $9=70 \%$ of votes)

\section{Conclusion}

Accurate diagnosis of ILDs is more important than ever, since treatment of IPF substantially differs from that of other ILDs. Evidence suggests that formal MDD among experts in the field of ILD improves the accuracy of IPF diagnoses. Whenever possible, patients should be referred to an ILD reference center for diagnosis and treatment initiation. Patients should be registered in national registries of cohort studies to monitor treatment effects and complications on a large scale in Switzerland. Off-label use and combination therapies should only be considered in a clinical study setting at this time. The choice of treatment currently depends on various parameters including patients' preferences and individual tolerance to side effects.

\section{Acknowledgements}

The authors thank Dr. Sabina Berezowska (Pathology, Bern University Hospital, Switzerland) for the histopathological images and Dr. M. Grob (pulmonologist, Biel, Switzerland) for careful reading of the manuscript.

\section{References}

1 Raghu G, et al: An official ATS/ERS/JRS/ ALAT statement: idiopathic pulmonary fibrosis: evidence-based guidelines for diagnosis and management. Am J Respir Crit Care Med 2011;183:788-824.

2 Raghu G, et al: An official ATS/ERS/JRS/ ALAT clinical practice guideline: treatment of idiopathic pulmonary fibrosis. An update of the 2011 clinical practice guideline. Am J Respir Crit Care Med 2015;192:e3-e19.

3 Behr J, et al: German guideline for diagnosis and management of idiopathic pulmonary fibrosis (in German). Pneumologie 2013;67: 81-111.

4 Cottin V, et al: French practical guidelines for the diagnosis and management of idiopathic pulmonary fibrosis. From the National Reference and the Competence centers for rare diseases and the Societe de Pneumologie de Langue Francaise (in French). Rev Mal Respir 2013;30:879-902.

5 Xaubet A, et al: Review of IPF diagnosis and management recommendations in Europe. Sarcoidosis Vasc Diffuse Lung Dis 2013;30: 249-261.

6 Baumann MH, et al: Management of spontaneous pneumothorax: an American College of Chest Physicians Delphi consensus statement. Chest 2001;119:590-602.

7 Geiser T: Idiopathic pulmonary fibrosis - a disorder of alveolar wound repair? Swiss Med Wkly 2003;133:405-411.
8 Funke M, Geiser T: Idiopathic pulmonary fibrosis: the turning point is now! Swiss Med Wkly 2015;145:w14139.

9 Walsh SL, et al: Relationship between fibroblastic foci profusion and high resolution CT morphology in fibrotic lung disease. BMC Med 2015;13:241.

$10 \mathrm{Kim}$ EJ, et al: Usual interstitial pneumonia in rheumatoid arthritis-associated interstitial lung disease. Eur Respir J 2010;35:1322-1328.

11 Morell F, et al: Chronic hypersensitivity pneumonitis in patients diagnosed with idiopathic pulmonary fibrosis: a prospective casecohort study. Lancet Respir Med 2013;1:685694

12 Song JW, et al: Pathologic and radiologic differences between idiopathic and collagen vascular disease-related usual interstitial pneumonia. Chest 2009;136:23-30.

13 Coultas DB, et al: The epidemiology of interstitial lung diseases. Am J Respir Crit Care Med 1994;150:967-972.

14 Baumgartner KB, et al: Cigarette smoking: a risk factor for idiopathic pulmonary fibrosis. Am J Respir Crit Care Med 1997;155:242248.

15 Savarino E, et al: Gastro-oesophageal reflux and gastric aspiration in idiopathic pulmonary fibrosis patients. Eur Respir J 2013;42: 1322-1331.
16 Baumgartner KB, et al: Occupational and environmental risk factors for idiopathic pulmonary fibrosis: a multicenter case-control study. Collaborating Centers. Am J Epidemiol 2000;152:307-315

17 Lee JS: The role of gastroesophageal reflux and microaspiration in idiopathic pulmonary fibrosis. Clin Pulm Med 2014;21:81-85.

18 Molyneaux PL, Maher TM: The role of infection in the pathogenesis of idiopathic pulmonary fibrosis. Eur Respir Rev 2013;22:376-381.

19 Wolters PJ, Collard HR, Jones KD: Pathogenesis of idiopathic pulmonary fibrosis. Annu Rev Pathol 2014;9:157-179.

20 Lee HL, et al: Familial idiopathic pulmonary fibrosis: clinical features and outcome. Chest 2005; 127:2034-2041.

21 Raghu G, et al: Idiopathic pulmonary fibrosis in US Medicare beneficiaries aged 65 years and older: incidence, prevalence, and survival, 2001-2011. Lancet Respir Med 2014;2: 566-572.

22 Nalysnyk L, et al: Incidence and prevalence of idiopathic pulmonary fibrosis: review of the literature. Eur Respir Rev 2012;21:355-361.

23 Karakatsani A, et al: Epidemiology of interstitial lung diseases in Greece. Respir Med 2009; 103:1122-1129.

24 Rudd RM, et al: British Thoracic Society Study on cryptogenic fibrosing alveolitis: response to treatment and survival. Thorax 2007;62:62-66. 
25 Collard HR, et al: Acute exacerbation of idiopathic pulmonary fibrosis. An International Working Group report. Am J Respir Crit Care Med 2016;194:265-275.

26 Collard HR, et al: Acute exacerbations of idiopathic pulmonary fibrosis. Am J Respir Crit Care Med 2007;176:636-643.

27 Ryerson CJ, et al: Acute exacerbation of idiopathic pulmonary fibrosis: shifting the paradigm. Eur Respir J 2015;46:512-520.

28 Peljto AL, et al: Association between the $M U C 5 B$ promoter polymorphism and survival in patients with idiopathic pulmonary fibrosis. JAMA 2013;309:2232-2239.

29 Noth I, et al: Genetic variants associated with idiopathic pulmonary fibrosis susceptibility and mortality: a genome-wide association study. Lancet Respir Med 2013;1:309-317.

30 Ley B, Brown KK, Collard HR: Molecular biomarkers in idiopathic pulmonary fibrosis. Am J Physiol Lung Cell Mol Physiol 2014; 307:L681-L691.

31 Ley B, et al: A multidimensional index and staging system for idiopathic pulmonary fibrosis. Ann Intern Med 2012;156:684-691.

32 Bahmer T, et al: The use of auto-antibody testing in the evaluation of interstitial lung disease (ILD) - a practical approach for the pulmonologist. Respir Med 2016;113:80-92.

33 Fischer A, et al: An official European Respiratory Society/American Thoracic Society research statement: interstitial pneumonia with autoimmune features. Eur Respir J 2015;46: 976-987.

34 Meyer KC, et al: An official American Thoracic Society clinical practice guideline: the clinical utility of bronchoalveolar lavage cellular analysis in interstitial lung disease. Am J Respir Crit Care Med 2012;185:1004-1014.

35 Welker L, et al: Predictive value of BAL cell differentials in the diagnosis of interstitial lung diseases. Eur Respir J 2004;24:10001006.

36 Casoni GL, et al: Transbronchial lung cryobiopsy in the diagnosis of fibrotic interstitial lung diseases. PLoS One 2014;9:e86716.

37 Tomassetti S, et al: Bronchoscopic lung cryobiopsy increases diagnostic confidence in the multidisciplinary diagnosis of idiopathic pulmonary fibrosis. Am J Respir Crit Care Med 2016;193:745-752.

38 Johannson KA, et al: Diagnostic yield and complications of transbronchial lung cryobiopsy for interstitial lung disease. A systematic review and metaanalysis. Ann Am Thorac Soc 2016;13:1828-1838.

39 Travis WD, et al: An official American Thoracic Society/European Respiratory Society statement: update of the international multidisciplinary classification of the idiopathic interstitial pneumonias. Am J Respir Crit Care Med 2013;188:733-748.

40 Walsh SL, et al: Multicentre evaluation of multidisciplinary team meeting agreement on diagnosis in diffuse parenchymal lung disease: a case-cohort study. Lancet Respir Med 2016;4:557-565.
41 Walsh SL, et al: Interobserver agreement for the ATS/ERS/JRS/ALAT criteria for a UIP pattern on CT. Thorax 2016;71:45-51.

42 Oldham JM, et al: TOLLIP, MUC5B and the response to $\mathrm{N}$-acetylcysteine among individuals with idiopathic pulmonary fibrosis. Am J Respir Crit Care Med 2015;192:1475-1482.

43 Raghu G, et al: Comorbidities in idiopathic pulmonary fibrosis patients: a systematic literature review. Eur Respir J 2015;46:11131130.

44 Fulton BG, Ryerson CJ: Managing comorbidities in idiopathic pulmonary fibrosis. Int $\mathrm{J}$ Gen Med 2015;8:309-318.

45 Seeger W, et al: Pulmonary hypertension in chronic lung diseases. J Am Coll Cardiol 2013; 62(suppl):D109-D116.

46 Lancaster LH, et al: Obstructive sleep apnea is common in idiopathic pulmonary fibrosis. Chest 2009;136:772-778.

47 Idiopathic Pulmonary Fibrosis Clinical Research Network, Raghu G, et al: Prednisone, azathioprine, and $\mathrm{N}$-acetylcysteine for pulmonary fibrosis. N Engl J Med 2012;366: 1968-1977.

48 Conte E, et al: Effect of pirfenidone on proliferation, TGF- $\beta$-induced myofibroblast differentiation and fibrogenic activity of primary human lung fibroblasts. Eur J Pharm Sci 2014; 58:13-19.

49 Noble PW, et al: Pirfenidone in patients with idiopathic pulmonary fibrosis (CAPACITY): two randomised trials. Lancet 2011;377: 1760-1769.

50 King TE Jr, et al: A phase 3 trial of pirfenidone in patients with idiopathic pulmonary fibrosis. N Engl J Med 2014;370:2083-2092.

51 Valeyre D, et al: Comprehensive assessment of the long-term safety of pirfenidone in patients with idiopathic pulmonary fibrosis. Respirology 2014;19:740-747.

52 King CS, Nathan SD: Practical considerations in the pharmacologic treatment of idiopathic pulmonary fibrosis. Curr Opin Pulm Med 2015;21:479-489.

53 Borie R, et al: Pharmacological management of IPF. Respirology 2016;21:615-625.

54 Wollin L, et al: Mode of action of nintedanib in the treatment of idiopathic pulmonary fibrosis. Eur Respir J 2015;45:1434-1445.

55 Hostettler KE, et al: Anti-fibrotic effects of nintedanib in lung fibroblasts derived from patients with idiopathic pulmonary fibrosis. Respir Res 2014;15:157.

56 Richeldi L, et al: Efficacy and safety of nintedanib in idiopathic pulmonary fibrosis. N Engl J Med 2014;370:2071-2082.

57 Richeldi L, et al: Nintedanib in patients with idiopathic pulmonary fibrosis: combined evidence from the TOMORROW and INPULSIS $^{\circledR}$ trials. Respir Med 2016;113:74-79.

58 Rochwerg B, et al: Treatment of idiopathic pulmonary fibrosis: a network meta-analysis. BMC Med 2016;14:18.
59 Canestaro WJ, et al: Drug treatment of idiopathic pulmonary fibrosis: systematic review and network meta-analysis. Chest 2016;149: 756-766.

60 Costabel U, et al: Efficacy of nintedanib in idiopathic pulmonary fibrosis across pre-specified subgroups in INPULSIS ${ }^{\circledR}$. Am J Respir Crit Care Med 2016;193:178-185.

61 Harari S, et al: Efficacy of pirfenidone for idiopathic pulmonary fibrosis: an Italian real life study. Respir Med 2015;109:904-913.

62 Raghu G, et al: Effect of nintedanib in subgroups of idiopathic pulmonary fibrosis by diagnostic criteria. Am J Respir Crit Care Med 2017;195:78-85.

63 Moodley Y, et al: Do all patients with idiopathic pulmonary fibrosis warrant a trial of therapeutic intervention? A pro-con perspective. Respirology 2015;20:389-394.

64 Loeh B, et al: Intraindividual response to treatment with pirfenidone in idiopathic pulmonary fibrosis. Am J Respir Crit Care Med 2015;191:110-113.

65 Wuyts WA, et al: First data on efficacy and safety of nintedanib in patients with idiopathic pulmonary fibrosis and forced vital capacity of $\leq 50 \%$ of predicted value. Lung 2016 ; 194:739-743.

66 Wiggins J, Strickland B, Turner-Warwick M: Combined cryptogenic fibrosing alveolitis and emphysema: the value of high resolution computed tomography in assessment. Respir Med 1990;84:365-369.

67 Wells AU, et al: Lone cryptogenic fibrosing alveolitis: a functional-morphologic correlation based on extent of disease on thin-section computed tomography. Am J Respir Crit Care Med 1997;155:1367-1375.

68 Doherty MJ, et al: Cryptogenic fibrosing alveolitis with preserved lung volumes. Thorax 1997;52:998-1002.

69 Mejia M, et al: Idiopathic pulmonary fibrosis and emphysema: decreased survival associated with severe pulmonary arterial hypertension. Chest 2009;136:10-15.

70 Akagi T, et al: Coexistent emphysema delays the decrease of vital capacity in idiopathic pulmonary fibrosis. Respir Med 2009;103: 1209-1215.

71 Ryerson CJ, et al: Clinical features and outcomes in combined pulmonary fibrosis and emphysema in idiopathic pulmonary fibrosis. Chest 2013;144:234-240.

72 Kohashi Y, et al: Clinical impact of emphysema evaluated by high-resolution computed tomography on idiopathic pulmonary fibrosis diagnosed by surgical lung biopsy. Respiration 2016;92:220-228.

73 Cottin V, et al: Combined pulmonary fibrosis and emphysema (in French). Presse Med 2007;36(pt 2):936-944.

74 Lancaster L, et al: Safety of pirfenidone in patients with idiopathic pulmonary fibrosis: integrated analysis of cumulative data from 5 clinical trials. BMJ Open Respir Res 2016; 3:e000105.
Idiopathic Pulmonary Fibrosis in Switzerland
Respiration 2017;93:363-378 DOI: $10.1159 / 000464332$ 
75 Nathan SD, et al: Effect of continued treatment with pirfenidone following clinically meaningful declines in forced vital capacity: analysis of data from three phase 3 trials in patients with idiopathic pulmonary fibrosis. Thorax 2016;71:429-435.

76 Milger K, et al: Switching to nintedanib after discontinuation of pirfenidone due to adverse events in IPF. Eur Respir J 2015;46:12171221.

77 Ogura T, et al: Safety and pharmacokinetics of nintedanib and pirfenidone in idiopathic pulmonary fibrosis. Eur Respir J 2015;45:13821392.

78 Idiopathic Pulmonary Fibrosis Clinical Research Network, Martinez FJ, et al: Randomized trial of acetylcysteine in idiopathic pulmonary fibrosis. N Engl J Med 2014;370: 2093-2101.

79 Behr J, et al: Safety and tolerability of acetylcysteine and pirfenidone combination therapy in idiopathic pulmonary fibrosis: a randomised, double-blind, placebo-controlled, phase 2 trial. Lancet Respir Med 2016;4:445-453.

80 Weill D, et al: A consensus document for the selection of lung transplant candidates: 2014 - an update from the Pulmonary Transplantation Council of the International Society for Heart and Lung Transplantation. J Heart Lung Transplant 2015;34:1-15.

81 Brown AW, Kaya H, Nathan SD: Lung transplantation in IIP: a review. Respirology 2016; 21:1173-1184.

82 https://www.cdc.gov/vaccines/schedules/ hcp/imz/adult-conditions.html.

83 Kenn K, Gloeckl R, Behr J: Pulmonary rehabilitation in patients with idiopathic pulmonary fibrosis - a review. Respiration 2013;86: 89-99.
84 Bahmer T, et al: Clinical correlates of reduced physical activity in idiopathic pulmonary fibrosis. Respiration 2016;91:497-502.

85 Frank RC, et al: Ambulatory oxygen in idiopathic pulmonary fibrosis: of what benefit? Eur Respir J 2012;40:269-270.

86 Bräunlich J, et al: Effects of nasal high flow on ventilation in volunteers, COPD and idiopathic pulmonary fibrosis patients. Respiration 2013;85:319-325.

87 Blivet S, et al: Outcome of patients with idiopathic pulmonary fibrosis admitted to the ICU for respiratory failure. Chest 2001;120: 209-212.

88 Rush B, et al: The use of mechanical ventilation in patients with idiopathic pulmonary fibrosis in the United States: a nationwide retrospective cohort analysis. Respir Med 2016; 111:72-76.

89 Yokoyama T, et al: Noninvasive ventilation in acute exacerbation of idiopathic pulmonary fibrosis. Intern Med 2010;49:1509-1514.

90 Vianello A, et al: Noninvasive ventilation in the event of acute respiratory failure in patients with idiopathic pulmonary fibrosis. J Crit Care 2014;29:562-567.

91 Lindell KO, et al: Palliative care and location of death in decedents with idiopathic pulmonary fibrosis. Chest 2015;147:423-429.

92 Ahmadi Z, et al: End-of-life care in oxygendependent ILD compared with lung cancer: a national population-based study. Thorax 2016;71:510-516.

93 Antoniou KM, Wells AU: Acute exacerbations of idiopathic pulmonary fibrosis. Respiration 2013;86:265-274.
94 Papiris SA, et al: Survival in Idiopathic pulmonary fibrosis acute exacerbations: the non-steroid approach. BMC Pulm Med 2015;15:162.

95 Raghu G, et al: Pulmonary hypertension in idiopathic pulmonary fibrosis with mild-tomoderate restriction. Eur Respir J 2015;46: 1370-1377.

96 Bayer Corporation: Bayer terminates phase II study with riociguat in patients with pulmonary hypertension associated with idiopathic interstitial pneumonias. 2016. http:// www.prnewswire.com/news-releases/ bayer-terminates-phase-ii-study-withriociguat-in-patients-with-pulmonaryhypertension-associated-with-idiopathicinterstitial-pneumonias-300267616.html.

97 Funke M, Geiser T, Schoch OD: Pulmonary hypertension associated with chronic lung diseases. Swiss Med Wkly 2016;146:w14363.

98 Kreuter M, et al: Screening for Helicobacter pylori in idiopathic pulmonary fibrosis lung biopsies. Respiration 2016;91:3-8.

99 Ghebre YT, Raghu G: Idiopathic pulmonary fibrosis: novel concepts of proton pump inhibitors as antifibrotic drugs. Am J Respir Crit Care Med 2016;193:1345-1352.

100 Lee JS, et al: Anti-acid treatment and disease progression in idiopathic pulmonary fibrosis: an analysis of data from three randomised controlled trials. Lancet Respir Med 2013;1:369-376.

101 Kreuter M, et al: Antacid therapy and disease outcomes in idiopathic pulmonary fibrosis: a pooled analysis. Lancet Respir Med 2016; 4:381-389.

$102 \mathrm{https} / / /$ clinicaltrials.gov/ct2/results?term $=$ azithromycin+and+ipf\&Search=Search. 